\title{
Plaid Cymru (1925-1979) : nationalisme gallois et dévolution
}

Plaid cymru (1925-1979): welsh nationalism and devolution

\section{Claire Charlot}

\section{CpenEdition}

Journals

Édition électronique

URL : http://journals.openedition.org/rfcb/1172

DOI : $10.4000 /$ rfcb. 1172

ISSN : 2429-4373

Éditeur

CRECIB - Centre de recherche et d'études en civilisation britannique

Édition imprimée

Date de publication : 2 janvier 2006

Pagination : 85-105

ISBN : 2-911580-23-0

ISSN : 0248-9015

Référence électronique

Claire Charlot, « Plaid Cymru (1925-1979) : nationalisme gallois et dévolution », Revue Française de Civilisation Britannique [En ligne], XIV-1 | 2006, mis en ligne le 15 octobre 2016, consulté le 19 avril 2019. URL : http://journals.openedition.org/rfcb/1172 ; DOI : 10.4000/rfcb.1172

\section{(c) (i) (9)}

Revue française de civilisation britannique est mis à disposition selon les termes de la licence Creative Commons Attribution - Pas d'Utilisation Commerciale - Pas de Modification 4.0 International. 


\title{
Plaid Cymru (1925-1979) : nationalisme gallois et dévolution
}

\author{
Claire CHARLOT
}

Université de Haute Bretagne (Rennes 2)

Fondé le 5 août 1925, le Parti national gallois (Plaid Genedlaethol Cymru) est à l'origine un groupe de pression dont la mission consiste à défendre le patrimoine linguistique et culturel gallois. En 1945, il décide de prendre un nouveau départ afin d'entamer une véritable conquête politique du pays de Galles, d'autant que son attitude pacifiste pendant la guerre l'a rendu parfois impopulaire ${ }^{1}$. Il se donne un nouveau nom (Plaid Cymru), se dote d'un nouveau président (Gwynfor Evans) ${ }^{2}$, et renforce son organisation au niveau local.

Les objectifs du parti sont clairement énoncés dans un opuscule (Plaid Cymru and Wales) ${ }^{3}$ rédigé par son président, qui réclame un parlement pour le pays de Galles et exhorte les Gallois à adhérer à son parti pour défendre la cause de l'autonomie locale (self-government), face à la mainmise d'un "gouvernement impérial », à savoir anglais. Pour Gwynfor Evans, autonomie signifie liberté, au niveau de l'individu et de la "communauté », terme clé dans la définition de l'idéologie nationaliste galloise: 'Freedom for Wales means freedom to realise her possibilities as a Welsh community; freedom for the Welshman means freedom to realise his possibilities as a Welshman in a free Welsh society. Freedom for the nation is a condition of freedom for the individual person. ${ }^{4}$ En dépit de résultats électoraux inégaux, de 1929 à $1966^{5}$, l'ambition de Plaid Cymru est de réveiller, voire d'éveiller, en chaque Gallois un sentiment d'allégeance politique à la nation galloise (Welsh nationhood), d'abord par le biais d'une appartenance linguistique et culturelle puis par le biais d'une socialisation à la défense des intérêts gallois. Mais la revendication politique d'appartenance à une nation ou ce que l'on pourrait qualifier d'«identité nationaliste» («être Gallois militant») ne coïncide pas nécessairement avec le sentiment d'une identité nationale (« se sentir Gallois») et

\footnotetext{
${ }^{1}$ Gwynfor Evans, secrétaire honoraire du mouvement pacifiste gallois (Heddychwyr Cymru) avait été objecteur de conscience comme nombre de ses camarades nationalistes. Le Parti travailliste, en revanche, s'était totalement impliqué dans l'effort de guerre puisqu'il avait participé au gouvernement de coalition dirigé par Winston Churchill. Cela lui vaudra de gagner, lors de l'élection législative du 5 juillet 1945, 25 sièges au pays de Galles sur 35. Plaid Cymru ne remportera aucun siège et n'enregistrera que 16017 voix.

${ }^{2}$ Il sera président du parti du $1^{\mathrm{er}}$ août 1945 au 31 octobre 1981.

${ }^{3}$ Gwynfor EVANS, Plaid Cymru and Wales, Swansea: Llyfraw'r Dryw, 1950, 73 p. L'éditeur avait demandé à chacun des dirigeants des partis gallois de rédiger un livre présentant son parti. Seul Gwynfor Evans s'exécuta.

${ }^{4}$ Idem, p. 10 et p. 16.

${ }^{5}$ Voir le Tableau 1, en annexe, qui résume la progression électorale de Plaid Cymru.
} 
Plaid Cymru devra redoubler d'efforts pour rendre crédible auprès de l'électorat gallois un programme nationaliste qui, au départ, s'adresse essentiellement à une élite intellectuelle soucieuse de préserver son patrimoine culturel.

\section{Quelle identité nationale galloise ?}

Définir une identité, a fortiori nationale, reste un exercice difficile et de nombreux sociologues se sont déjà interrogés sur la façon dont le sentiment identitaire se construit et change en fonction de données personnelles à l'individu. Concernant le pays de Galles, plusieurs questions se posent. Tout d'abord, comment isoler une identité galloise d'une identité britannique ? L'une prime-t-elle sur l'autre ou peuvent-elles être concomitantes ? $?^{6}$ Comment, d'ailleurs, réussir à définir des critères objectifs de l'identité, qu'elle soit galloise ou britannique ? Ces questions sont essentielles pour un parti qui, comme Plaid Cymru, prône l'adhésion à des valeurs galloises dites authentiques (érigées en Welsh credentials) et cherche également à convaincre un nombre croissant de Gallois de le rejoindre sur le terrain nationaliste de leur sauvegarde à tout prix. Plaid Cymru considère que les critères déterminants d'une identité galloise (Welsh identity) sont la langue (galloise), la religion (non-conformiste) et l'origine géographique (être natif du pays de Galles ou avoir des ancêtres gallois).

La défense de la langue galloise a très tôt joué un rôle prépondérant dans les revendications nationalistes. Comme l'écrivent Harold Carter et John Aitchinson, le caractère et la vitalité d'une culture dépendent dans une large mesure de la langue, qui aide à perpétuer les traditions et à influencer les modes de perception et de conduite $^{7}$. Or si l'on examine la proportion de la population capable de parler le gallois, on constate qu'elle passe de la moitié en 1901 (49,9\%) à moins d'un tiers en $1951(28,9 \%)$, pour tomber à moins d'un cinquième en $1981(18,9 \%)^{8}$. On note à cet égard un différentiel important entre les générations ${ }^{9}$.

Du point de vue de la distribution géographique des Gallois susceptibles d'adhérer aux valeurs traditionnelles, on peut distinguer différentes zones en

\footnotetext{
${ }^{6}$ Voir notamment l'introduction de John OSMOND ('Coping with a Dual Identity', pp. xixxlvi) à son ouvrage The National Question Again. Welsh Political Identity in the 1980s, Llandysul: Gomer Press, 1985.

7 'Language areas and language change in Wales: 1961-1981', in : I. HUME \& W.T.R. PRYCE, (eds.), The Welsh and their Country, Llandysul: Gomer Press/OU, 1986. p. 1.

${ }^{8}$ Chiffres tirés des différents recensements. Une question sur la pratique du gallois a été posée à chaque recensement depuis 1891. Aujourd'hui, la proportion de galloisants est remontée à un cinquième $(21 \%)$ en 2001 grâce à la création d'écoles galloises. Pour la première fois en 2001, une question supplémentaire a été introduite pour connaître la proportion de personnes comprenant le gallois. La compréhension du gallois était légèrement supérieure en 2001 à sa pratique orale $(24 \%)$. Il faut d'ailleurs distinguer ici entre différentes compétences linguistiques : la langue orale $(21 \%)$, la lecture $(20 \%)$ et l'écriture $(18 \%)$. Seuls $16 \%$ de Gallois possédaient les trois compétences en 2001.

${ }^{9}$ En 2001, les deux cinquièmes des enfants de 10 à 15 ans (39\%) savent parler, lire et écrire le gallois, contre un quart des adolescents de 16 à 19 ans (25\%), un dixième des adultes de 35 à 49 ans $(11 \%)$ et un sixième de ceux de plus de 75 ans $(15 \%)$ ONS, Focus on Wales: Its People, London: TSO, janvier 2004, p. 5.
} 
fonction de la proportion d'habitants galloisants. En 1964, au moment où renaissent les nationalismes régionaux, E.G. Bowen montre, lors d'une conférence radiodiffusée (BBC Wales), que le pays de Galles se divise en deux, pour des raisons de topographie, entre un pays de Galles traditionnel (Inner Wales) et un pays de Galles anglicisé (Outer Wales). Le premier, protégé au nord et à l'ouest par un terrain accidenté, a gardé une culture authentique : une langue galloise associée à une pratique religieuse, d'abord liée au catholicisme puis au renouveau spirituel méthodiste du dix-huitième siècle. Le second, au sud et à l'est, s'est ouvert très tôt à l'influence anglaise et puritaine en raison de l'accès relativement facile aux vallées et aux plaines. Au dix-huitième siècle, l'Église d'Angleterre (de type high church) y était bien implantée. E.G. Bowen en conclut que c'est la culture du nord et de l'ouest qui a donné au pays «sa personnalité, sa langue, sa religion et son chant » et qu'il s'agit là du « vrai pays de Galles » ${ }^{10}$. Cette vision est à nuancer aujourd'hui ${ }^{11}$ mais on trouve toujours au nord du pays, notamment dans les régions de Gwynedd et d'Ynys Môn, les plus fortes concentrations de galloisants : respectivement $61 \%$ et $51 \%$ lors du recensement de 2001. Du point de vue de l'origine des Gallois, au moment du recensement de 1951, $83 \%$ des personnes vivant au pays de Galles y étaient nées. Le pourcentage a diminué au fil des ans mais est resté conséquent : $77 \%$ en $1991,75 \%$ en $2001^{12}$. Confortant cette hypothèse de l'importance de la terre et de la langue, Denis Balsom a utilisé les résultats d'une enquête réalisée à l'été 1979 (Welsh Election Study) afin d'examiner si, à l'époque du référendum, on pouvait parler de sentiment identitaire. À la question 'Do you normally consider yourself to be Welsh, British, English or something else ?', $57 \%$ de l'électorat gallois avaient répondu Gallois, $34 \%$ Britannique, $8 \%$ Anglais ${ }^{13}$. Le tri croisé langue/identité fait apparaître non plus deux communautés mais trois groupes sociolinguistiques, décrits sous le nom de «modèle des trois Galles » par Balsom : le pays de Galles galloisant à l'identité galloise (au nord et à l'Ouest : Y Fro Gymraeg), le pays de Galles gallois mais non-galloisant (Welsh Wales - le sud du pays de Galles) et enfin le pays de Galles britannique non-galloisant et qui ne se sent pas gallois (British Wales) ${ }^{14}$.

On voit bien ici le défi que s'est lancé Plaid Cymru puisque, contrairement à leurs homologues écossais, les nationalistes gallois ont toujours intégré une

\footnotetext{
${ }^{10}$ Conférence reproduite in : I. HUME \& W.T.R. PRYCE, (eds.), op. cit., p. 85. '...its personality, its language, its religion and song. These survive into the modern epoch and represent the real Wales.'

${ }^{11}$ Christopher G.A. BRYANT, The Nations of Britain, Oxford: O.U.P., 2006. 'Voicing Wales', pp. 117-156.

${ }^{12}$ Focus on Wales, op. cit., p. 5.

${ }^{13}$ Denis BALSOM, 'The Three-Wales Model', in : John OSMOND (ed.), op. cit., pp. 2-3. L'enquête a cependant été réalisée après l'échec du référendum, ce qui a sans doute eu une influence sur les résultats.

${ }^{14}$ Idem, p. 6. Y Fro Gymraeg : Caernarfon, Carmarthen, Ceredigion and Pembroke North, Conwy, Meirionydd Nant Conwy, Ynys Môn. Welsh Wales : Aberavon, Blaenau Gwent, Caerphilly, Cynon Valley, Gower, Islwyn, Llanelli, Merthyr Tydvil and Rhymney Neath, Ogmore, Pontypridd, Rhondda, Swansea East, Swansea West, Torfaen. British Wales : Alyn and Deeside, Brecon and Radnor, Bridgend, Cardiff Central, Cardiff North, Cardiff South and Penarth, Cardiff West, Clwyd North West, Clwyd South West, Delyn, Monmouth, Montgomery, Newport East, Newport West, Pembroke, Vale of Glamorgan, Wrexham.
} 
dimension culturelle, et en particulier linguistique, à leur définition de l'identité nationale galloise. Le problème reste que les non-galloisants (non-Welsh speakers) peuvent éprouver un sentiment identitaire, d'appartenance à une nation, sans pour autant considérer que la langue galloise constitue une condition nécessaire à leur adhésion affective, voire politique, au pays de Galles. En bref, un sentiment identitaire n'implique pas forcément un sentiment nationaliste, surtout s'il se détermine avant tout en fonction d'une pratique linguistique en voie de disparition. Gwynfor Evans avait déjà pris la mesure du problème dans les années 1950 puisque, dans son premier ouvrage, il définissait le « test de nationalité gallois » comme celui de l'appartenance à une «communauté galloise» et non celui d'une revendication linguistique ou généalogique ${ }^{15}$. Cette tentative de récupérer des votes hors des bastions traditionnels galloisants échoua dans une large mesure puisque les Gallois restèrent très méfiants vis-à-vis de l'offre politique nationaliste. Aux élections législatives de 1950 et 1951, Plaid Cymru ne réussit pas à gagner de circonscription et son score électoral stagna puis recula, passant de $1,2 \%$ des suffrages à $0,8 \%$. Pour progresser, le Parti nationaliste gallois devrait jouer sur la notion d'une histoire commune, d'un vécu gallois qui puissent transcender les différences en termes de langue, d'origine et surtout d'identification partisane, en raison de la forte influence politique des libéraux puis des travaillistes après $1945^{16}$.

\section{Le réveil du nationalisme gallois et la montée de Plaid Cymru}

Après les lois d'union de 1536 et $1542^{17}$, le pays de Galles fut totalement intégré à l'Angleterre et c'est au XIX $\mathrm{X}^{\mathrm{e}}$ siècle, avec le réveil des nationalismes régionaux, que certaines initiatives furent prises pour éveiller le sentiment national gallois. Déjà s'opposaient au pays de Galles une bourgeoisie galloisante, nonconformiste, libérale et une classe de propriétaires anglicisés et anglicans. La religion et la langue galloise avaient été érigées en symboles de la résistance à l'influence anglaise. Le résultat fut l'émergence de deux dynamiques parallèles, et sans lien entre elles, qui allaient faire le lit du mouvement nationaliste naissant.

La première dynamique est d'ordre politique et concerne les activités, en 1886, d'un mouvement politique d'obédience libérale, Cymru Fydd (Wales of the Future), qui prône la politique de l'autonomie (Home Rule for Wales - également appelée Domestic self-government) et du désétablissement de l'Église d'Angleterre au pays

\footnotetext{
15 'To-day, the majority of these immigrants [English people], or their descendants, are proud to call themselves Welshmen; and since common membership of the Welsh community rather than language or descent is the test of nationality in Wales, nationalists are proud to know them as fellow-Welshmen. Wales has no finer patriots than some who have no knowledge of the national tongue, whereas many who speak the language think they thereby fulfil their duty to Wales; Welshmen who "hide behind the language" are unfortunately a common phenomenon in our land.' Plaid Cymru and Wales, op. cit., p. 17.

${ }^{16}$ Voir les tableaux des résultats en annexe.

${ }^{17}$ Le pays de Galles avait d'abord été annexé à l'Angleterre en 1284 (Statutum Walliae ou Statute of Rhuddlan) par ordonnance royale, puis il avait été incorporé définitivement par les lois «d'union » de 1536 et 1542 (Laws in Wales Acts). La loi de 1536 prévoyait l'élection de 26 membres gallois au Parlement anglais ; celle de 1542 couvrait les questions de propriété terrienne, de droit et de justice. Dans le préambule de la loi de 1536, il était déjà fait mention d'une langue spécifique au pays de Galles.
} 
de Galles. En janvier 1888, le groupe publie un journal, du même nom, et commence à créer toute une structure de sections locales qui se fédèrent, au niveau régional, en août 1894 à Llandrindod Wells ${ }^{18}$. Si le mouvement est appelé à disparaître une dizaine d'années plus tard, l'idée de l'autonomie du pays de Galles va cependant faire son chemin.

La seconde dynamique est d'ordre culturel et concerne la mise en place d'institutions spécifiquement galloises, faisant naître un sentiment de fierté chez ceux qui en ont connaissance. Ce sont la création (ou plus exactement la résurrection) d'un festival annuel des arts, l'Eisteddfod, en 1880 ; la fondation de l'Université du pays de Galles en 1893 et celle de la Bibliothèque nationale en 1907 ; ou plus importante d'une certaine façon, la naissance de la Fédération de la jeunesse galloise (Urdd Gobaith Cymru) en $1922^{19}$. Comme l'écrit Kenneth O. Morgan, cette dernière joua un rôle crucial dans l'éveil d'une « conscience nationale non partisane $»^{20}$. La renaissance intellectuelle constituait la prémisse d'une renaissance politique puisque c'est dans le contexte de l'Eisteddfod national, à Pwllheli, qu'est créé Plaid Cymru en 1925, en raison du fait qu'une élite éduquée a pris conscience du déclin de la langue galloise et de la culture qui y est associée. Dès sa fondation, le Parti nationaliste fait appel à l'imaginaire gallois en projetant le mythe du gwerin, ce peuple de Gallois hérö̈ques qui, bravant l'oppresseur, se sont révoltés et ont revendiqué leur identité nationale ${ }^{21}$.

\section{Les revendications politiques de Plaid Cymru}

Le Parti nationaliste gallois annonce, dès sa fondation, trois objectifs, qui restent inchangés en $1969^{22}$ :

1) To secure self-government for Wales.

2) To safeguard the culture, language, traditions and the economic life of Wales.

\footnotetext{
18 Voir Kenneth O. MORGAN, Rebirth of a Nation. A History of Modern Wales, Oxford/Cardiff: OUP/UWP, 1981. p. 115.

${ }^{19} \mathrm{La}$ Fédération avait été créée par Ifan ab Owen Edwards afin d'inculquer aux jeunes l'amour de leur langue maternelle et de leur culture. En 1927, le mouvement comptait 5000 membres ; en 1934, 50 000. La fédération a fondé la première école galloise en 1939.

${ }^{20}$ Idem, p. 252. 'But the main impact of the Urdd was in capturing the imagination of the young, in stimulating a positive, non-nationalistic love of country and of its literary and artistic heritage, in providing the youth of Wales with the kind of educational, cultural and moral inspiration so much needed in the swirling tides of post-war Britain and the years of depression.'

${ }^{21}$ Voir à ce sujet la contribution de David Adamson, 'The intellectual and the National Movement in Wales', in: Ralph FEVRE \& Andrew THOMPSON, Nation, Identity and Social Theory. Perspectives from Wales, Cardiff: UWP, 1999. pp. 48-68.

${ }^{22}$ Le logo de Plaid Cymru qui a été adopté en 1933, le Triban représentant trois montagnes vertes, est censé refléter ces trois objectifs. Les montagnes incarnent également les valeurs de l'unité et de l'indépendance puisqu'elles ont combattu, dans le folkore gallois, avec les princes contre les rois anglais et ont garanti au pays une intégrité territoriale. Voir E. Gwynn MATTHEWS, This Is Plaid Cymru, Cardiff: Plaid Cymru, 1969. p. 25.
} 
3) To secure for Wales the right to become a member of the United Nations. $^{23}$

La première revendication n'est pas tout à fait nouvelle dans la mesure où, à plusieurs reprises, des propositions de loi touchant à l'autonomie locale du pays de Galles ont été présentées au Parlement, mais sans succès ${ }^{24}$. Elle effraie cependant les électeurs, malgré les tentatives de Saunders Lewis (Président du parti de 1926 à 1939) de rassurer les Gallois sur ce point :

First of all, let us not ask for independence for Wales. Not because it is impracticable, but because it is not worth having [...] it is a cruel and material thing leading to violence and oppression and ideas that have been proved wrong [...] We demand, therefore, not independence but freedom, and the meaning of freedom in this matter is responsibility. ${ }^{25}$

Le deuxième objectif est certain de toucher l'électorat des terres galloisantes du nord et de l'ouest du pays mais le Parti nationaliste a bien du mal à être pris au sérieux ailleurs lorsqu'il réclame une dévolution des pouvoirs, d'autant que l'identité galloise est loin de coïncider à l'époque avec une identité nationaliste et que le parti est perçu comme un groupuscule radical qui s'agite à la gauche des travaillistes.

Le troisième objectif fait partie des ambitions internationalistes de Plaid Cymru dans la veine des mouvements socialistes. Plaid Cymru est d'ailleurs le seul parti au pays de Galles à revendiquer un rôle dans les relations internationales. En 1968, Gwynfor Evans suggère la création d'un « Conseil des États britanniques » ou «Confédération britannique » (Britannic Confederation) sur le modèle du Conseil nordique, créé en $1952^{26}$. Le corollaire serait la création d'un « Marché Commun » entre le pays de Galles et l'Angleterre.

Ces trois objectifs sont restés des constantes du programme nationaliste même si certaines formulations ont changé ou ont été adaptées en fonction de la nouvelle donne politique nationale et internationale. La défense de la langue galloise, prioritaire en 1925, a été reléguée au deuxième plan, voire déléguée à d'autres institutions au moment où pourtant les revendications nationalistes se font plus pressantes, dans les années 1960. La protection du patrimoine linguistique gallois reste néanmoins l'une des revendications affichées du parti car il s'agit de la seule institution galloise à avoir traversé les âges ${ }^{27}$. Le parti prône donc une politique de

\footnotetext{
${ }^{23}$ Idem, p. 24.

${ }^{24}$ Plusieurs Government of Wales Bills ont été présentées en 1914, 1922, 1954 et 1967. Il y a également eu un colloque sur la dévolution en 1920 (Speaker's Conference). En 1914, par exemple, la proposition de loi (qui n'a pas dépassé le stade de la première lecture) a été «sponsorisée » par un riche industriel gallois, E.T. John, qui avait fait fortune dans l'acier à Middlesborough.

${ }^{25}$ Egwyddorion Cenedlaetholdeb, 1926, p. 4. Cité in : E.G. Matthews, op. cit., p. 4.

${ }^{26}$ Welsh Nation, décembre 1968.

${ }^{27}$ Ce qui fait écrire à Gwynfor EVANS, op. cit., p. 126 : 'It's a grave mistake to measure the success of Plaid Cymru in electoral terms alone. As well as being a political party, it's a national movement that revivifies the nation as it works from within it, gathering together
} 
bilinguisme. Le statut spécifique du Gallois est d'ailleurs reconnu en 1967 dans un rapport officiel sur l'enseignement primaire au pays de Galles, rapport comparable au Rapport Plowden de la même époque en Angleterre. Il sera possible pour chaque enfant d'apprendre sa langue maternelle (anglaise ou galloise) dès la maternelle, la deuxième langue étant apprise à l'école primaire. Mais chaque parent sera libre de décider ce qu'il souhaite pour son enfant. Le rapport Gittins avait en effet montré que $10 \%$ des parents trouvaient que l'apprentissage du Gallois constituait un handicap pour leurs enfants et $35 \%$ que cela ne leur apportait rien de plus.

Since it is the Welsh language which in large measure gives Wales its own peculiar identity and carries an important part of its historical tradition, it has a claim on the loyalty of those who claim to be Welsh. We have the right to be what we are and the responsibility of ensuring that we hand down what has been given us. ${ }^{28}$

Fort de ses objectifs, le Parti nationaliste va exploiter la fibre identitaire en montrant à deux reprises, en 1935 et 1955, que des instances politiques ont porté gravement atteinte à l'intégrité de la terre galloise. Ces violations du territoire gallois réussiront à éveiller un sentiment de sympathie vis-à-vis des nationalistes qui verront, à chaque occasion, leur score électoral augmenter.

\section{L'intégrité territoriale}

La première atteinte à l'intégrité territoriale du pays de Galles intervient dans un contexte où Plaid Cymru est électoralement quasi inexistant ${ }^{29}$. Mais le parti provoque un intérêt certain pour la cause nationaliste lorsqu'en 1936 trois de ses membres ${ }^{30}$ mettent le feu aux bâtiments qui se trouvent sur le terrain que vient d'acquérir la Royal Air Force à Pen-y-Berth (péninsule de Llyn) dans le Caernarfonshire. Faisant fi des susceptibilités galloises, le gouvernement a en effet décidé d'implanter une zone de manœuvres militaires sur un site associé à Owain Glyn Dwr, le héros gallois, et comble de la provocation, les travaux ont commencé exactement quatre cents ans après la Loi d'union avec l'Angleterre. À la suite de leur geste de défiance vis-à-vis du gouvernement, les trois incendiaires se rendent volontairement à la police qui les défère alors à la justice. Un premier jury gallois ne parvenant pas à les condamner, le procès est transféré à Londres (Old Bailey) où les accusés sont condamnés à des peines de prison de neuf mois à Wormwood Scrubs pour avoir refusé de parler anglais. Ce fait de bravoure accroît la popularité de Plaid, considéré comme une victime du gouvernement britannique (« anglais » diraient

those who dearly wish to ensure a national future for Wales. That is the most fundamental difference between Plaid Cymru and the British parties.' La place à accorder à la langue dans le programme de Plaid Cymru a été vivement débattue dans le parti dans les années soixante et soixante-dix.

${ }^{28}$ Primary Education in Wales and Monmouthshire (Gittins Report), London: HMSO, août 1967, p. 213.

${ }^{29}$ Aux législatives de 1929, Lewis Valentine obtient 609 voix. En 1931 et 1935, les scores ne dépassent pas 2600 voix, au mieux.

${ }^{30}$ Il s'agit de Saunders Lewis, le président du parti (1926-1939), D.J. Williams, ancien mineur, et du Révérend Lewis Valentine, un pasteur baptiste. 
certains). Mais l'embellie est de courte durée et l'attitude pacifiste du parti pendant la guerre les prive de nombreux sympathisants. L'événement reste néanmoins célèbre dans les annales du parti sous le nom du « feu de Llyn ». Il sert également de précédent en matière de violence organisée et préméditée, en cas de force majeure. Le futur chef de parti de Plaid, Gwynfor Evans, se déclare, à l'inverse, en faveur d'un mouvement de non-violence dans le cadre du fonctionnement démocratique des institutions britanniques. Il condamnera, par exemple, les incendies criminels perpétrés contre des résidences secondaires anglaises au pays de Galles à partir de 1979.

La deuxième atteinte à l'intégrité territoriale du pays a lieu bien après la guerre, en 1955, lorsque la ville de Liverpool annonce son intention de créer un réservoir d'eau au pays de Galles, entraînant ainsi la destruction de la communauté de Capel Celyn puisqu'il était prévu d'inonder la vallée de Tryweryn (Cwm Tryweryn). Malgré l'opposition virulente de la plupart des députés gallois, une loi créant le réservoir (Llyn Celyn) est adoptée par le Parlement mais la disparition d'une vallée dans le Meirionedd a frappé les esprits et le parti enregistre de nouveaux soutiens lors des élections de 1959, plus de 2 points de pourcentage de voix supplémentaires par rapport à 1955 . Les résultats sont particulièrement bons à Caernarfon (20\%) et à Rhondda West (17\%).

Les deux événements, très différents de nature, ont ceci en commun qu'ils suscitèrent chez les Gallois le sentiment qu'on ne pouvait toucher impunément à leur terre. Cette résistance à l'intrus anglais est restée gravée dans l'inconscient collectif gallois et nombre de références sont faites encore aujourd'hui à ces deux événements. Cela ne signifie pas pour autant, à la veille du mouvement pour la dévolution, l'émergence d'une force nationaliste organisée et Gwynfor Evans décrit ainsi la situation dans les années d'après-guerre :

In those days it was ridiculous to think of living for Wales and completely unimaginable that anyone should be prepared to die for it. Welsh nationality belonged to the periphery of life. It occurred to none save a small band of Nationalists that this nation should rule itself. They didn't dream that the national entity of Wales should be the basis of economic, political, and social life and that there should be a Welsh state. ${ }^{31}$

\section{L'amorce d'un mouvement politique pour la dévolution}

Dans ses mémoires, Gwynfor Evans affirme que c'est Plaid Cymru qui eut le premier l'idée de créer un mouvement - Parliament for Wales Campaign - pour une dévolution administrative accrue. En 1949, la campagne commence par une série de réunions politiques annuelles (rallies). Le premier slogan utilisé à Machynlleth est celui d' "un Parlement gallois dans les cinq ans ». Le modèle est celui du Covenant Movement écossais. La campagne est officiellement lancée sous les auspices du mouvement culturel (Undeb Cymru Fydd) car les organisateurs

${ }^{31}$ Gwynfor EVANS. For The Sake of Wales. The Memoirs of Gwynfor Evans. Translated by Meic Stephens. Cardiff: Welsh Academic Press, 1996 (second edition 2001), p. 133. 
souhaitent lui donner plus de poids, et un comité d'organisation est élu, avec à sa présidence Megan Lloyd George ${ }^{32}$. Le mouvement est pluripartite mais les libéraux, sauf Megan Lloyd George, refusent généralement de prendre la parole en public pour soutenir la campagne. Ce n'est pas le cas de la minorité de députés travaillistes engagés dans le mouvement ${ }^{33}$ mais ceux-ci sont cependant vivement critiqués par leur parti et seul S.O. Davies (député de Merthyr Tydfil) restera impliqué jusqu'à la fin de la campagne puisqu'en mars 1955, il introduit une proposition de loi (rédigée par Dewi Watkin Powell, l'un des avocats amis des nationalistes) pour un Parlement gallois. La campagne se limitera essentiellement au recueil de signatures pendant plusieurs années et Goronwy Roberts présentera au Parlement une pétition d'environ 250000 signatures. Après quoi, la campagne cessa d'elle-même en 1956. Malgré un côté assez laborieux, la campagne réussit cependant à lancer l'idée, chez certains, qu'une autonomie locale du pays de Galles serait souhaitable. L'élection de 1955 permit d'ailleurs de voir la progression des nationalistes, même si le nombre de votes global restait dérisoire (45 000) au regard des autres partis.

Fort de ce succès, Plaid Cymru ne reste pas inactif. Deux initiatives lui donnent l'occasion de participer à une campagne de désobéissance civile. Dans le premier cas, le parti apporte son soutien à Trefor et Eileen Beasley de Llangennech qui ont refusé d'acquitter leurs impôts locaux à Llanelli car le formulaire n'était rédigé qu'en anglais. Plaid Cymru rachètera le mobilier des époux, qui avait été saisi par la municipalité. De même, lorsque Gwynfor Evans sollicite son homonyme Gwynfor S. Evans pour se présenter aux élections municipales de Y Betws, en avril 1958, ce dernier n'a plus de formulaires de candidature en langue anglaise à sa disposition. Il remet donc une liste en gallois à l'agent préposé aux élections, qui la refusa. Plaid Cymru fait appel de la décision en Haute cour de justice à Londres et Dewi Watkin Powell gagnera le procès. La municipalité n'aura pas le droit d'interjeter appel et à partir de ce moment-là tous les documents électoraux (formulaires, affiches...), pour les campagnes locales et nationales, pourront être rédigés à la fois en anglais et en gallois.

Mais le catalyseur qui aide finalement Plaid Cymru à obtenir son premier siège à Westminster est, paradoxalement, un discours radiodiffusé de l'ancien président du parti, Saunders Lewis ${ }^{34}$, qui en février 1962, dénonce la disparition probable de la langue galloise et appelle à sa sauvegarde plutôt qu'à l'organisation d'un mouvement pour la dévolution. Plaid Cymru ne souhaite pas s'associer de trop près à ce discours mais lors de son université d'été à Pontarddulais, une association est créée pour la défense de la langue (Cymdeithas yr Iaith Gymraeg - Welsh Language Society). Ceci va permettre au Parti nationaliste de continuer à mener un combat politique sans que lui soit reproché une politique linguistique et culturelle sectaire, limitée aux seules personnes parlant le gallois, c'est-à-dire concentrées dans le nord et l'ouest du pays. Les liens entre l'association et les nationalistes resteront

\footnotetext{
${ }^{32}$ Megan Lloyd George représentait alors la circonscription d'Ynys Môn (Anglesey) et elle était vice-présidente du Parti libéral. Le secrétaire était Dafydd Miles. Le comité comprenait, entre autres personnes, William George, le frère de Lloyd George et Dafydd Jenkins, qui avait organisé la pétition pour la défense de la langue galloise avant-guerre.

33 S.O. Davies, Tudor Watkins, Cledwyn Hughes, Goronwy Roberts, T.W. Jones.

${ }^{34}$ Tynged yr Iaith (The Fate of the Language).
} 
néanmoins très étroits. Leur combat, ainsi que celui d'autres associations galloises, conduira à l'adoption du Welsh Language Act de $1967^{35}$.

Le 14 juillet 1966, suite au décès de Megan Lloyd George, Gwynfor Evans réussit à se faire élire député lors de l'élection législative partielle de Carmarthen ${ }^{36}$. Comme il l'évoque dans ses mémoires, la date était hautement symbolique : traité signé entre Owain Glyn Dwr et le roi de France en 1404, chute de la Bastille en 1789. Mais surtout, les nationalistes prouvent pour la première fois qu'ils sont devenus une alternative crédible au niveau des circonscriptions galloises. La part du vote nationaliste a augmenté de 16,1\% à $39 \%$ des suffrages exprimés. Avec cette victoire, le Parti nationaliste gallois montre qu'il ne mérite plus le sobriquet de «petit parti ( plaid bach) et qu'il faut désormais compter avec lui ${ }^{37}$. À partir de 1970, Plaid Cymru présentera des candidats dans toutes les circonscriptions galloises. Le nationalisme avait le vent en poupe puisqu'en novembre 1967 Winifred Ewing remportait en Écosse la partielle de Hamilton ${ }^{38}$.

\section{Un contexte favorable aux nationalistes}

Les deux campagnes référendaires de 1979 et 1997 ne sont absolument pas comparables si l'on considère le rôle joué par Plaid Cymru. La première campagne s'inscrit dans un contexte de progression des voix nationalistes (après les succès de février et d'octobre 1974) mais aussi d'incertitude liée à la façon dont est envisagé le processus de dévolution législative et exécutive par les travaillistes, pris au dépourvu par la montée des nationalismes en Grande-Bretagne. C'est en effet pendant l'examen du projet de loi sur l'Écosse et le pays de Galles ${ }^{39}$ qu'il est décidé de procéder à un référendum, d'abord envisagé comme délibératif puis, sous la pression de l'opposition, comme consultatif. Les consultations référendaires en Écosse et au pays de Galles seront organisées le même jour, le $1^{\text {er }}$ mars, jour symbolique de la Saint David au pays de Galles. Plaid Cymru, pourtant en position de force, adoptera une attitude extrêmement prudente comme si le parti sentait que l'opinion galloise ne le suivrait pas sur le terrain d'une plus grande dévolution. Le deuxième

\footnotetext{
${ }^{35}$ La loi, votée sous les travaillistes (avec le soutien des conservateurs), intègrera les conclusions de la Commission Hughes Parry (Report on the Legal Status of the Welsh Language, London: HMSO, Cmnd. 2785, 1967) que Keith Joseph, conservateur, avait nommée après la publication d'un livre blanc en novembre 1963 sur le sujet.

${ }^{36}$ Partielle : Gwynfor Evans (Plaid Cymru) : 16179 voix ; G. Prys Davies (travailliste) : 13743 ; D.H. Davies (libéral) : 8650 ; S.J. Day (conservateur) : 2934 soit une majorité de 2436 voix pour le parti nationaliste. Lors des élections législatives, le 31 mars 1966, Gwynfor Evans arrive en troisième position, avec 7416 voix, derrière les candidats travailliste (Lady Megan Lloyd George : 21 221) et libéral (D.H. Davies : 11 988).

${ }^{37}$ Dans un article publié au lendemain de la victoire de Carmarthen, E. Hudson Davies souligne que pour continuer sur sa lancée, Plaid Cymru devra décider de la place à accorder à la langue galloise dans son programme '...no party can hope to continue fighting on a different platform in different constituencies' (p. 331) et présenter une politique économique « cohérente ». Voir 'Welsh Nationalism', Political Quarterly, Vol. 39, 1968, pp. 322-332.

${ }^{38}$ Winifred Ewing a remporté la partielle, en novembre 1967, avec une majorité de 1798 voix.

${ }^{39}$ Le projet de loi The Scotland and Wales Bill a été introduit au Parlement en décembre 1976.
} 
référendum, en 1997, est organisé dans un tout autre contexte. Les travaillistes, avec Tony Blair, se sont totalement impliqués dans un processus de réforme constitutionnelle, dont la dévolution législative et exécutive fait partie, et le pays de Galles devra se prononcer une semaine après l'Écosse, le gouvernement comptant bien sur l'effet d'entraînement du vote écossais pour influencer les indécis gallois. Plaid Cymru s'engagera à fond dans cette campagne afin d'obtenir enfin l'autonomie, même limitée, que lui offrent les travaillistes.

Lors des deux décennies qui précèdent le référendum, plusieurs facteurs ont amené un sursaut nationaliste qui s'est concrétisé avec l'arrivée au Parlement de deux représentants de Plaid Cymru en février 1974 et trois en octobre de la même année $^{40}$. Ces facteurs sont de deux ordres : politique et économique. D'un point de vue politique, ce sont paradoxalement les concessions des différents gouvernements, des années 1950 à 1970, qui alimentent les revendications nationalistes. Le processus de dévolution administrative a commencé avec l'éducation puis s'est poursuivi après la guerre, à chaque fois que les pressions nationalistes se faisaient sentir. En février 1949, dans un document officiel (The Conservative Policy for Wales and Monmouthshire), le Parti conservateur affirme que si l'Angleterre et le pays de Galles constituent à eux deux une seule entité économique, on doit néanmoins reconnaître que le pays de Galles est une unité nationale et qu'il faut donc envisager la création d'un ministre chargé de le représenter au Cabinet (Cabinet Minister). Il sera assisté d'un Conseil (Council of Wales). Cette démarche s'inscrivait bien dans l'exercice d'une dévolution administrative dans la mesure où le pays de Galles gagnait une certaine autonomie sans que l'on touche pour autant aux prérogatives du pouvoir central. Lors de la campagne électorale de 1950, Winston Churchill avait d'ailleurs déclaré :

If I thought a Welsh Parliament at the present time would be in the best interests of the Welsh people, I would not hesitate to recommend it to you. But Wales and England, though two nations, are a single economic whole. A Welsh Parliament which could not consider the main economic issues, or could consider them only in isolation from English views, would not be good for you, or for us. ${ }^{41}$

Les conservateurs s'engagèrent néanmoins à œuvrer pour le pays de Galles, sans doute persuadés qu'ils calmeraient ainsi toute velléité d'autonomie et qu'ils y trouveraient un avantage électoral aux dépens des travaillistes. À la suite des promesses électorales de 1951, un secrétaire d'état aux Affaires galloises (Minister for Welsh Affairs) est nommé mais ses fonctions s'ajoutent à celles du ministre de l'Intérieur, en octobre 1951, puis à celles du ministre du Logement et des Collectivités locales, dès janvier 1957. L'aboutissement logique du processus est la création, en avril 1960, d'une nouvelle commission parlementaire à la Chambre des communes, le Welsh Grand Committee, ainsi que l'annonce par le Premier ministre, Harold

${ }^{40}$ En février 1974, Dafydd Wigley est élu à Caernarfon et Dafydd Elis Thomas à Meirionydd. Gwynfor Evans est battu de trois voix à Carmarthen par le candidat travailliste sortant mais il gagne le siège avec 3640 voix d'avance en octobre 1974. Ses collègues conservent leurs sièges à Caernarfon et Meirionydd.

${ }^{41}$ Discours de Cardiff, 8 février 1950. 
Macmillan, le 5 mars 1963, d'un ministère des Affaires galloises (Welsh Office). Les travaillistes parachèveront le processus de dévolution administrative en transférant les fonctions ministérielles touchant au pays de Galles à un ministre des Affaires galloises, rattaché au Cabinet (Secretary of State for Wales), à partir d'octobre 1964.

Paradoxalement, la façon dont les affaires galloises sont alors traitées par les différents ministres ainsi que la politique d'ouverture culturelle qui a été menée, loin d'apaiser les revendications nationalistes les encouragent. Les lois destinées à protéger, voire promouvoir, la langue galloise (comme le Welsh Language Act de 1967) incitent les Gallois à se pencher sur leur passé et à retrouver, avec le sens de l'histoire, un intérêt pour l'autonomie et les réflexes identitaires.

En raison de la montée des nationalistes en Écosse et au pays de Galles, le gouvernement Wilson nomme, en 1969, une Commission royale chargée de faire des propositions sur la Constitution. Celle-ci rend son rapport en $1973^{42}$. Un chapitre entier est consacré au pays de Galles et les rapporteurs constatent à ce propos : 'The concern for democracy in Wales seems to be particularly strong in Wales. This strength of feeling shows itself in the widespread demand for an elected Welsh assembly, and in the vigorous criticism of appointed ad hoc bodies. ${ }^{, 43}$ Ils pensent par ailleurs que le pays de Galles, comme l'Écosse, doit être doté d'une assemblée élue au suffrage direct mais ils ne s'accordent pas sur les pouvoirs à déléguer à une telle institution. Six membres se déclarent favorables à une dévolution législative, trois à une assemblée délibérative et consultative pour le pays de Galles (dont un pour des assemblées similaires en Écosse et au pays de Galles), et deux à une dévolution exécutive au pays de Galles et en Écosse ${ }^{44}$.

D'un point de vue économique, la situation du pays de Galles est aggravée par le fait qu'elle repose pour l'essentiel sur des industries traditionnelles : industries minières (charbon, étain) et sidérurgie. En 1979, les aciéries emploient presque la moitié $(43 \%)$ de la population active du secteur public et contribuent, à hauteur de $35 \%$, à la production de l'acier dans le pays. Mais le nombre de sidérurgistes a considérablement diminué à Port Talbot, Llanwern et Shotton (qui cessera d'ailleurs son activité en 1980). Quant aux puits de mines, ils ferment eux aussi, les uns après les autres (perte de 50000 mineurs depuis la guerre). Le résultat, malgré l'aide des différents gouvernements (avec notamment la création de la Welsh Development Agency et du Development Board for Rural Wales en 1976), est un taux de chômage très élevé, le deuxième du Royaume-Uni derrière l'Irlande du Nord.

\footnotetext{
${ }^{42}$ Royal Commission on the Constitution 1969-1973 (Kilbrandon Commission), Vol. I Report, London: HMSO, Cmnd. 5460, octobre 1973.

${ }^{43}$ Idem, pp. $33-45$ : p. 16 , para. 376.

${ }^{44}$ Idem, p. 336, para 1123. Dans l'ordre, il s'agit de Lord Kilbrandon, Mr Davies, Sir Mark

Henig, Dr Longmuir, Professor Newark, Sir Ben Bowen Thomas ; Professor Street et Mrs

Trenaman (Sir David Renton) ; Lord Foot et Sir James Steel.
} 


\section{Plaid Cymru en campagne : le Réferendum de 1979}

Lorsque la proposition d'un référendum est annoncée, le 10 février $1977^{45}$, les adversaires de la dévolution se réjouissent de laisser à l'opinion le soin de décider pour eux du sort qu'ils réservent à ce nouvel enjeu politique. Le terrain a été bien préparé avec la publication de documents officiels sur le sujet ${ }^{46}$. Cela n'empêche cependant pas le premier projet de loi d'échouer et le gouvernement Callaghan est alors contraint de négocier avec les libéraux un nouveau projet de loi sur la dévolution afin de s'assurer leur soutien, sans lequel il se trouvera mis en minorité à la Chambre.

La nouvelle Loi sur le pays de Galles introduit une dévolution minimale puisqu'elle ne concerne que les enjeux sociaux ou environnementaux ${ }^{47}$. Il est précisé aux Articles 28 et 29 que les sujets touchant aux relations internationales feront toujours partie du domaine réservé de l'État britannique. Aucune dévolution exécutive n'est envisagée si ce n'est à travers le travail des commissions de la nouvelle Assemblée (Articles 17 et 18). En effet, il est prévu que l'Assemblée galloise aura 80 représentants (chaque circonscription envoyant deux personnes excepté les circonscriptions les plus peuplées qui en enverront trois au départ). Les affaires seront gérées par un ensemble de commissions dont le travail sera coordonné par un Comité exécutif sous la présidence d'un Chef exécutif (Chief Executive). Il est également prévu qu'un commissaire parlementaire (Ombudsman) et qu'un Contrôleur général (Comptroller and Auditor-General) soient nommés.

Dans l'attente d'une date précise pour l'organisation du référendum, et donc bien avant le début de la campagne officielle de 1979, Plaid Cymru élabore sa stratégie électorale. Dans une note confidentielle, aujourd'hui déposée aux archives nationales galloises, Ewrfyl ap Gwilym évoque les différents problèmes qui se posent au parti ${ }^{48}$. Le Parti nationaliste gallois se trouve placé dans une situation difficile. Il souhaite d'un côté faire campagne pour la dévolution mais trouve que la Loi sur le pays de Galles ne va pas assez loin dans la voie d'une autonomie

\footnotetext{
${ }^{45}$ Ceci fut fait suite à un amendement au projet de loi lors de son examen en commission. L'amendement avait été proposé par Leo Abse, deputé de Pontypool qui demandait l'insertion au projet de loi d'un article prévoyant l'organisation d'un référendum sur la question de la dévolution. Pour une discussion des débats à la Chambre, voir l'ouvrage de Vernon BOGDANOR, Devolution in the United Kingdom, Oxford: OUP, 1979 (second edition 2001). ${ }^{46}$ Our Changing Democracy: Devolution to Scotland and Wales, London: HMSO, 27 novembre 1975, Cmnd. 6348 (White Paper) et Devolution to Scotland and Wales: Supplementary Statement, London: HMSO, août 1976, Cmnd. 6585.

${ }^{47}$ Le projet de loi Wales Bill est introduit en novembre 1977 au Parlement. Le premier vote (2nd reading) a lieu le 15 novembre 1977 (295 voix contre 264), le deuxième (3rd reading) par 292 voix contre 264, le 9 mai 1978. Pour les prérogatives de la future Assemblée, voir les Articles 10 et 11, p. 6, de la Wales Act 1978 (Chapter 52): '...museums, art galleries, libraries, the Welsh language, the arts, crafts, sport and other cultural and recreative activities'; '...services for the war disabled and ... grants towards the carrying on of public passenger transport undertakings in Wales.'

${ }^{48}$ Eurfyl ap Gwilym, Plaid Cymru and the Referendum, confidential draft (Plaid Cymru archives - L5), janvier 1977.
} 
galloise $^{49}$; par ailleurs, il ne souhaite pas faire du référendum une campagne personnelle de façon à éviter l'amalgame du mouvement pour la dévolution et du mouvement nationaliste chez les électeurs.

Ayant constaté après l'expérience de la campagne référendaire sur l'Europe de 1975 que la mobilisation des militants et de la direction du parti était plus efficace lorsque les objectifs étaient préalablement définis, le conseiller insiste sur la nécessité de partir unis en campagne, sans doute fort de l'expérience du dernier référendum. En effet, la campagne contre l'Europe, lors du référendum de 1975, s'était soldée par un échec pour le parti, qui s'était divisé sur le slogan «L'Europe, Oui ; la CEE Non ». Les électeurs, dans tout le pays de Galles, avaient souhaité continuer à adhérer à l'Europe et même dans la région de Gwynedd, ils avaient voté Oui à 70,6\%. Les électeurs avaient donc infligé une défaite cuisante aux nationalistes qui avaient fait campagne pour le Non. Dafydd Wigley avait, dès le départ, refusé de participer à la campagne.

Pour la campagne du référendum, d'un point de vue tactique, il s'agit d'évaluer quand la campagne de Plaid Cymru doit commencer et où. D'un point de vue stratégique, il convient de cibler les adversaires reconnus et d'entretenir un climat favorable à l'idée que la dévolution est voulue par l'ensemble des Gallois et non pas seulement par les nationalistes. D'autant que certains de leurs membres ne partagent pas du tout l'enthousiasme pour une Assemblée aux pouvoirs restreints. Le référendum est donc l'occasion pour Plaid Cymru de faire avancer la cause du nationalisme tout en faisant croire à l'électorat qu'il est divisé sur l'enjeu de la dévolution (comme semblent l'accréditer les journalistes) :

Except in certain key areas the Assembly proposals must not be identified with us, they need to be identified with the moderate mainstream of Welsh political and public life [...] ... to the public at large the impression that Plaid Cymru is lukewarm in supporting the Assembly needs to be cultivated. ${ }^{50}$

Sauf que, n'en déplaise au conseiller, la version donnée par les journalistes n'est pas si éloignée de la vérité. Depuis 1978, des dissensions internes agitent le Parti nationaliste sur l'opportunité de participer au mouvement pour le Oui. Plaid Cymru est entré en campagne auprès des travaillistes, sans grand enthousiasme et sous la pression de son leader, Gwynfor Evans. Or la cellule de réflexion que celuici a créée au sein du parti ainsi que le conseil exécutif du parti avaient décidé, en 1977, que Plaid Cymru s'opposerait à tout référendum sur la dévolution ${ }^{51}$. Il fallut tout le doigté et la force de persuasion de Gwynfor Evans, qui avait jugé bon de passer outre cette décision, pour amener le parti à s'impliquer dans le mouvement pour le Oui. Durant la campagne la tension entre les travaillistes et Plaid Cymru est

\footnotetext{
${ }^{49}$ Idem, p. 5. 'Plaid Cymru has always made it clear that we consider the powers of the Assembly inadequate and that our aim is full self-government for Wales within the European Community.'

${ }^{50}$ Idem, pp. 9-10.

${ }^{51}$ Laura McALLISTER, Plaid Cymru -The Emergence of a Political Party, Bridgend: Seren, 2001, pp. 132-133.
} 
tangible. John Morris (député d'Aberafan) prévient d'ailleurs qu'il ne parlera à la tribune, lors du lancement de la campagne à Carmarthen, qu'à la condition que Gwynfor Evans ne soit pas à ses côtés ${ }^{52}$.

Pour le conseiller de Plaid Cymru, il faut que le discours du parti s'adapte en fonction des publics: nationaliste ici, pro-dévolutionniste là. Il conviendra d'《 éduquer » les foules et non pas de s'adresser à des assemblées de sympathisants convaincus. D'où la nécessité pour le parti de se placer en retrait par rapport aux partisans reconnus de la dévolution, un choix tactique réfléchi. En ce qui concerne le déroulement de la campagne, Plaid Cymru a prévu d'entretenir un climat propice à la dévolution par voie de communiqués et de lettres afin de ne pas laisser la campagne du Non occuper tout le terrain. Mais la véritable offensive ne devra intervenir que quelques semaines avant le vote. 'There is a good case to hold back our main campaign until the last four to six weeks prior to the referendum. Too long a campaign could run out of steam, both from the point of view or arguments, and the energy of workers, the public would also get bored. ${ }^{53}$

L'aspect le plus intéressant de la stratégie électorale de Plaid Cymru reste la façon dont il envisage de faire campagne dans les différentes régions. Ainsi à Gwynedd, il fera appel au sentiment nationaliste et patriotique ; à Dyfed, il insistera sur la réforme impopulaire du gouvernement local ; dans le Glamorgan et les vallées de Gwent où se trouvent les groupes les plus hostiles à la dévolution (conservateurs, Confédération de l'industrie britannique, Front national, Associations des propriétaires terriens), il soulignera le fait que les députés travaillistes hostiles à l'Assemblée sont ceux qui préfèrent Chelsea à Pontypool, Kingston on Thames à Bedwellty $^{54}$; enfin à Clwyd, il faudra faire jouer le sentiment de la peur du chômage. L'idéal pour le parti serait que tous les adversaires de la dévolution soient assimilés aux conservateurs, présentés comme des réactionnaires face aux forces de progrès favorables à la dévolution (essentiellement à gauche). Plaid Cymru va donc s'associer à la campagne du Oui, de façon relativement discrète.

Toute une série de notes de synthèse scientifiques fait le point des enjeux de la campagne $^{55}$ et réfute les arguments des adversaires de la dévolution. Ces derniers dénoncent essentiellement le coût élevé d'une Assemblée, le fait qu'elle créera un niveau supplémentaire au niveau du gouvernement local et qu'elle portera atteinte à l'unité du Royaume-Uni.

À l'argument avancé avec force par les conservateurs (notamment Lord Hailsham) que le Royaume-Uni serait affaibli voire mis en danger, D. Elystan

\footnotetext{
${ }^{52}$ Gwynfor EVANS, op. cit., p. 218.

${ }^{53}$ Idem, p. 2.

${ }^{54}$ Idem, p. 7. Il s'agit d'une allusion aux députés gallois travaillistes, opposés à la dévolution : Leo Abse et Neil Kinnock. Au sein du Parti travailliste, on trouve un groupe de six irréductibles du sud du pays de Galles, opposés à la dévolution : Leo Abse, Neil Kinnock, Donald Anderson, Fred Evans, Ioan Evan, Ifor Davies.

${ }^{55}$ Ces documents sont déposés aux archives de Plaid Cymru, à la Bibliothèque nationale du pays de Galles, à Aberystwyth (Coll 9/12, L1-L5).
} 
Morgan répond que les adversaires confondent le concept d'uniformité avec celui d'unité :

To them there can be no unity for Britain unless the constituent parts which give it life and variety are reduced to a dull, grey conformity with its pulse regulated machine-like from the centre. They want to squeeze Britain into a constitutional and cultural strait-jacket. Such people totally fail to understand the creative energy which spring to life only on account of Britain being a 'community of communities. ${ }^{56}$

Il cite la mise en place d'institutions régionales en Europe de l'Ouest : en Allemagne, en Italie, en Suisse et plus récemment en Espagne. De façon intéressante, on trouve dans cette note de synthèse le même type d'argument que celui avancé au XIX ${ }^{\mathrm{e}}$ siècle pour l'élargissement du suffrage en 1831, à savoir que si le système politique souhaite conserver le respect et la loyauté du peuple gallois, il lui faut réformer le fonctionnement du Parlement. Face aux conservateurs qui agitent l'épouvantail du séparatisme et jouent sur la peur des électeurs, notamment en matière de chômage. Plaid Cymru se veut rassurant, affirmant que la dévolution, loin de désunir, raffermira l'union. D.E. Morgan ironise : 'It is almost as base an argument as the contention that it is dangerous to allow the people of Wales the right to vote in case they should exercise their franchise to establish a Welsh republic. ${ }^{57}$

Pendant la campagne, Plaid Cymru insiste en particulier sur ce qu'il appelle le « déficit démocratique » lié au fonctionnement des institutions mises en place par les différents gouvernements d'après-guerre. Au niveau parlementaire, les affaires galloises sont gérées sans que les députés gallois puissent réellement intervenir. Ainsi dans la session qui précède le référendum, en 1977-1978, les députés gallois n'ont eu que huit occasions de poser des questions au ministre des Affaires galloises et à ses secrétaires d'état, soit 3 heures et 20 minutes de questions orales sur toute une session. John Morris (Secretary of State for Welsh Affairs), ardent partisan de la dévolution, le concède d'ailleurs volontiers. Par ailleurs, sur 400 décisions prises par le gouvernement concernant le pays de Galles, seules 28 ont été débattues à la Chambre ou examinées par les députés ${ }^{58}$. La journée galloise (Welsh Day), instituée en 1944, reste symbolique, d'autant qu'il s'écoule parfois jusqu'à dix-huit mois, selon l'urgence des affaires parlementaires en cours, entre deux journées. Les adversaires de la dévolution ont bien proposé que soit renforcée la Grande commission aux affaires galloises mais Plaid Cymru objecte d'une part, que la commission n'a déjà pratiquement aucun pouvoir et, d'autre part, que la lourdeur de la tâche constituerait à elle seule un temps plein de parlementaire.

Au niveau régional, la bureaucratie est devenue intolérable et le déficit démocratique encore plus criant. Plaid Cymru accuse les quelque 2500 fonctionnaires du Welsh Office d'être frileux en matière d'innovation, ce qui nuit à

${ }^{56}$ D. Elystan MORGAN, The Welsh Assembly - Towards a New Unity in Britain, Referendum Study paper 4. (Plaid Cymru archives - L3), p. 1.

${ }^{57}$ Idem, p. 4.

${ }^{58}$ Idem, p. 2. 
la situation économique et sociale du pays de Galles. Pour le Parti nationaliste, il est clair qu'une Assemblée galloise semble être la panacée à tous les maux politiques gallois :

The 79 Assembly will open up this vast secret area of government, publicly air the problems involved, the policy alternatives available, scrutinise decisions, monitor the implementation of policies and make the existing governmental process in Wales more open and more directly accountable to the people of Wales. That is what devolution is essentially about. ${ }^{59}$

Mais c'est surtout aux organisations quasi-gouvernementales (quangos quasi-autonomous governmental organisations) que s'en prend Plaid Cymru. On compte à l'époque environ une soixantaine de ces organisations dont les membres sont nommés à la discrétion du gouvernement (en l'occurrence ici le Welsh Secretary of State). Il est estimé que ces organismes qui gèrent de fait les affaires galloises échappent totalement au contrôle local puisqu'ils ne doivent rendre des comptes qu'à celui qui les a constitués, le ministre des Affaires galloises. Leur rôle est loin d'être négligeable puisqu'en 1976-1977 ils ont dépensé la somme de 446 millions de livres sterling ${ }^{60}$. Sans compter qu'ont été nommés dans certains de ces organismes des candidats travaillistes malheureux aux élections législatives, provoquant ainsi l'ire de nombreux électeurs gallois. Plaid Cymru va exploiter ce mécontentement ainsi que celui lié à la réforme des collectivités locales galloises de 1974, après l'adoption de la Loi sur les collectivités locales (Local Government Act) de 1972.

Concernant le coût qu'entraînerait une Assemblée galloise, le Parti nationaliste fait valoir qu'une fois les dépenses initiales payées (environ 3,5 millions de livres), les dépenses de fonctionnement de l'Assemblée représenteront pour chaque contribuable moins d'un demi-penny par semaine. Plaid Cymru fait réellement le point sur tous les arguments avancés pour et contre la dévolution. La plupart de ces arguments, jusqu'aux mêmes exemples, sont repris dans la plate-forme électorale que présente la Wales For the Assembly Campaign pour le référendum du $1^{\mathrm{er}}$ mars $^{61}$.

\section{Un résultat accablant pour les nationalistes}

La campagne qui s'ouvre le 8 février et se termine le jour du vote, le $1^{\text {er }}$ mars 1979, est largement dominée par l'actualité écossaise et le Parti travailliste n'a fait aucun effort au pays de Galles pour expliquer aux électeurs le contenu des lois sur la dévolution. La couverture médiatique de la campagne galloise a été nettement

\footnotetext{
${ }^{59}$ Gwilym Prys DAVIES, The Assembly. Putting the People in Charge, Referendum Study Paper 2 (Plaid Cymru archives - L2), p. 1.

${ }^{60}$ Barry S. JONES, Gethin WILLIAMS, The Assembly. A Bargain for Wales, Referendum Study Paper (Plaid Cymru archives - L5), p. 2.

${ }^{61}$ Wales for the Assembly Campaign, Referendum Manifesto - Safeguarding Our Democratic Future, 1979, 6p. (Plaid Cymru archives - L4). Le document est organisé autour de six points: 'Introduction, Putting the people in charge, The financial cost and democratic benefit, The tangible benefits, The voice of Wales in a wider world, Conclusion.'
} 
inférieure à celle de la campagne écossaise. Si bien qu'à la veille du référendum, les résultats des enquêtes de sondages sont peu encourageants, voire alarmants, puisque le Non gagne peu à peu du terrain (voir Tableau 1).

Tableau 1 : Sondages réalisés avant le vote du $1^{\mathrm{er}}$ mars 1979 - résultats en pourcentages

\begin{tabular}{lllll}
\hline & BBC & HTV & BBC & BBC \\
& $(\mathbf{2 7 . 2 . 7 9 )}$ & $\mathbf{( 2 0 . 2 . 7 9 )}$ & $\mathbf{( 1 . 2 . 7 9 )}$ & $\mathbf{( 0 9 . 1 9 7 8 )}$ \\
Pour & 22.3 & 22 & 33 & 37.8 \\
Contre & 64.8 & 57 & 45.8 & 47.7 \\
NSP & 12.8 & 21 & 21.2 & 14.5 \\
\hline
\end{tabular}

Source: Paper from Conservative and Unionist Central Office Wales Area, Referendum Results, Chris Butler to the No Campaign movement, sent 'with compliments', 1979, 4 p. (No Campaign, Archives de la Bibliothèque nationale du pays de Galles, Aberystwyth).

La campagne du Non (No Assembly All Party Group) a été très active, en revanche, comme en témoigne la vaste correspondance déposée aux archives nationales du pays de Galles. Meurig Williams, l'attachée de presse du mouvement, écrit ainsi le 14 février 1979 à Eric Lambert (Ynys Môn) pour lui demander de trouver des personnes susceptibles de contrer les arguments en faveur de la dévolution, à l'occasion d'un débat télévisé organisé à Brangwyn Hall (Swansea), le 27 février, soit pratiquement à la veille du vote : 'Can you and would you please organise five fluent vociferous Welsh audience/questioners from your area - an unquestionably important one - if we are to counter the views of the Nationalist MP. ${ }^{62}$ Elle se plaint également de la couverture biaisée du Western Mail qui, pour favoriser le Oui, fait accroire que l'abstention est équivalente au Non ${ }^{63}$.

Le $1^{\text {er }}$ mars 1979, les Gallois se déclarent à 79,8\% (956 330 voix) contre le projet de loi sur la dévolution (soit 46,9\% des inscrits) contre 20,2 \% (243 048 voix) pour le projet (11,9\% des inscrits). Le taux de participation électorale est honorable pour un référendum puisqu'un Gallois sur deux s'est déplacé aux urnes $(58,8 \%)$. Mais le résultat est sans appel, même dans les circonscriptions galloisantes, et le projet est enterré. On trouve les taux d'électeurs favorables à la dévolution les plus élevés à Gwynedd $(34,4 \%)$ et à Dyfed $(28,1 \%)$, les plus faibles, sans surprise, à Gwent $(12,1 \%$ ) et dans le Glamorgan (South Glam. : 13,1\% ; West Glam. : 18,7 \% et Mid-Glam. : 20,2\% ${ }^{64}$. Il n'est pas même question de ratiociner sur le célèbre

\footnotetext{
${ }^{62}$ Il s'agit en l'occurrence de Dafydd Ellis Thomas, auprès de qui débattront trois autres députés: Denzil Denvies, Ifor Davies et Wyn Roberts (All Welsh Debate). (Archives nationales, No Assembly Campaign Papers 1, Jan.-March 1979).

${ }^{63}$ Lettre envoyée par Meurig Williams à Duncan Gardiner et G. Rich du Western Mail et du South Wales Echo, le 23 février 1979. (Ibid.).

${ }^{64}$ Par rapport aux enquêtes précédentes, le vote de Dyfed et de Gwynedd a augmenté alors qu'il s'est particulièrement effondré à Gwent et, dans une moindre mesure, dans dans le Glamorgan. Voir Chris BUTLER, op. cit., p. 4.
} 
amendement Cunningham ${ }^{65}$ qui avait fixé un seuil minimal de $40 \%$ des inscrits puisque le Non est massif ${ }^{66}$. Il y a eu quatre fois plus d'électeurs gallois défavorables que favorables à la proposition faite lors du référendum.

Dans leur étude des comportements électoraux des Gallois en 1979, des chercheurs $^{67}$ ont montré que si l'âge n'avait eu qu'une faible incidence sur le vote (les jeunes par exemple étant plus favorables à la dévolution mais également moins susceptibles d'aller voter que leurs aînés), l'identification partisane avait joué un rôle crucial. Plaid Cymru avait bien réussi à mobiliser ses troupes: seul 1 sympathisant sur $5(18 \%)$ n'était pas allé voter ; et ceux qui s'étaient déplacés aux urnes avaient voté Oui à $93 \%$. En revanche, la surprise venait du côté libéral puisque si une majorité de sympathisants avait voté $(74 \%)$, elle avait rejeté la proposition d'une assemblée à $83 \%$. Du côté conservateur, la mobilisation électorale avait été la même (74 \% de votants) mais comme on l'attendait le refus de la dévolution recueillait $90 \%$ des suffrages conservateurs exprimés. Quant aux travaillistes qui avaient proposé la réforme, ils n'avaient pas réussi à imposer une consigne de vote favorable à la dévolution. $63 \%$ avaient voté mais seulement $28 \%$ d'entre eux avaient approuvé la proposition. Le vote était sans doute autant un vote de protestation contre le gouvernement que de rejet de la proposition sur la dévolution ${ }^{68}$. Gwynfor Evans écrit ainsi dans ses mémoires :

The result was a rout from the point of view of Wales but a resounding success for the Labour and Conservative Parties. Wales was degraded in the sight of the world and humiliated in the eyes of its own people by the hugely negative result. Four times as many voted against an Assembly as voted in favour. Even in Gwynedd, where two of the M.P.s were Nationalists, there were twice as many against as in favour. That day, $1^{\text {st }}$ March 1979, went down as the blackest in the history of Wales. ${ }^{69}$

\footnotetext{
${ }^{65}$ L'amendement est ainsi appelé car il a été présenté par George Cunningham, lors de l'examen en commission de l'article 82(2) de la Loi sur l'Écosse. L'article concernait l'organisation d'un référendum et Cunningham lui apportait une modification en imposant un seuil minimal de $40 \%$ des inscrits pour que la Loi sur l'Écosse, une fois votée, entre en vigueur, faute de quoi la loi serait abrogée. L'amendement fut adopté par 166 voix contre 151, le 25 janvier 1978 et incorporé à l'Article 85(2) de la Loi sur l’Écosse de 1978. La même condition fut introduite pour la Loi sur le pays de Galles, à l'Article 80(2).

${ }^{66}$ Ibid. Chris Butler avait fait le savant calcul des votes qu'il faudrait obtenir pour parvenir à ce seuil de 40\%. Registre électoral gallois en février $1979: 2.083 .797$ électeurs, déduction faite des jeunes qui ne pourraient voter que le 2 mars : 2056 349. Estimation des décès de février au jour du vote : 14,900; des prisonniers : 800 ; des étudiants et personnel infirmier à une autre adresse : 2 600. À savoir 2038049 électeurs. Seuil de 40\%: 815220.

${ }^{67}$ Richard Wyn JONES, Dafydd TRYSTAN, Bridget TAYLOR, 'Voting patterns in the Referendum', in : J. Barry JONES, Denis BALSOM, The Road to the National Assembly for Wales, Cardiff: UWP, 2000.

${ }^{68}$ Idem, p. 169 (données des tableaux 10.6 et 10.7).

${ }^{69}$ Gwynfor EVANS, op. cit., p. 218. D'ailleurs les conservateurs obtiendront leur meilleur score électoral depuis 1874 au pays de Galles, remportant 11 sièges aux législatives de 1979.
} 
Il est intéressant de noter ici que le Parti travailliste est assimilé au Parti conservateur alors même que le premier était censé défendre la cause de la dévolution et le second la condamner.

\section{Conclusion}

La loi (Wales Act) sera donc abrogée le 26 juin 1979 par 191 voix contre 8. «L'hiver de tous les mécontentements ( (the Winter of Discontent) de 1978-1979 avait sans doute été de mauvais augure pour la suite des événements puisque les observateurs constataient qu'il était difficile de faire la part des choses entre un vote anti-Callaghan (Premier ministre depuis avril 1976) et un vote anti-dévolution. La série de grèves et de mouvements syndicaux avait profondément marqué les esprits à tel point que les conservateurs utilisèrent encore, pendant deux campagnes électorales au moins (1979 et 1983), les images liées aux grèves de cette époque.

Plaid Cymru fut durement touché par l'échec du référendum puisque le 3 mai 1979, lors des élections législatives nationales, il enregistra une nette baisse des suffrages par rapport aux dernières élections ( -2.7 points) et perdit au profit des travaillistes le siège de Carmarthen (celui de son leader), durement gagné en octobre 1974. Le parti connaît alors une traversée du désert dans les années 1980 mais les excès des politiques thatchériennes ${ }^{70}$ conduiront à l'élimination progressive des conservateurs au pays de Galles ${ }^{71}$. Sous la présidence de Dafydd Wigley ${ }^{72}$, Plaid Cymru prendra un nouvel élan et se lancera dans une nouvelle bataille pour la défense du patrimoine culturel, réclamant une quatrième chaîne de télévision, en gallois, ainsi qu'une parité officielle entre les deux langues (anglaise et galloise). Le succès à terme de ces deux revendications ${ }^{73}$ jettera les bases de la reconstruction du parti, comme en témoignent la victoire du Oui au référendum sur la dévolution organisé le 18 septembre 1997 et surtout la victoire éclatante de Plaid Cymru aux premières élections à l'Assemblée galloise en 1999.

\footnotetext{
${ }^{70}$ Les Gallois critiqueront en particulier les politiques économiques conduites par les conservateurs (tous les puits de mine seront fermés sauf un) et qui toucheront durement le pays de Galles ainsi que des décisions très controversées comme la nomination de ministres des Affaires galloises anglais et non d'origine galloise ou encore l'augmentation du nombre de quangos, à un tel point que l'on parle de quangocracy.

${ }^{71}$ Aux trois élections législatives de 1992, 1997 et 2001, les conservateurs ne gagneront aucun siège au pays de Galles.

${ }^{72}$ Président du parti de 1981 à 1984 puis de 1991 à 2000.

${ }^{73}$ Après la menace d'une grève de la faim de Gwynfor Evans, le gouvernement conservateur tiendra la promesse électorale de la création d'une chaîne télévisée galloise ( $44 \mathrm{C}$, Sianel Pedwar Cymru) qui commença à diffuser des émissions le 2 novembre 1982. Le Welsh Language Act de 1993, voté avec le concours de Wyn Roberts au ministère des Affaires galloises, établira la parité officielle entre les deux langues anglaise et galloise.
} 


\section{Annexes}

Tableau 1 : Plaid Cymru, 1945-1979 : Votes, candidats et élus au pays de Galles aux élections législatives

\begin{tabular}{|l|c|c|c|c|c|c|c|c|c|c|c|}
\hline & 1945 & 1950 & 1951 & 1955 & 1959 & 1964 & 1966 & 1970 & 19745 & 19740 & 1979 \\
\hline $\begin{array}{l}\text { Candidats } \\
\text { présentés }\end{array}$ & 7 & 7 & 4 & 11 & 20 & 23 & 20 & 36 & 36 & 36 & 36 \\
\hline $\begin{array}{l}\text { Sièges au } \\
\text { pays de } \\
\text { Galles }\end{array}$ & 35 & 36 & 36 & 36 & 36 & 36 & 36 & 36 & 36 & 36 & 36 \\
\hline Voix (\%) & 1,1 & 1,2 & 0,7 & 3,2 & 5,2 & 4,8 & 4,3 & 11,5 & 10,7 & 10,8 & 8,1 \\
\hline $\begin{array}{l}\text { Sièges } \\
\text { remportés }\end{array}$ & 0 & 0 & 0 & 0 & 0 & 0 & 0 & 2 & 2 & 3 & 2 \\
\hline
\end{tabular}

Sources : Données tirées des ouvrages de Peter JOYCE, Politico's Guide to UK General Elections 1932-2001, London: Politico's, 2004 et de Laura McALLISTER, op. cit., Table 1, p. 112.

Tableau 2 : État des forces au pays de Galles, 1945-1979 : élus des différentes formations politiques aux élections législatives

\begin{tabular}{|l|l|l|l|l|l|l|l|l|l|l|l|}
\hline & $\mathbf{1 9 4 5}$ & $\mathbf{1 9 5 0}$ & $\mathbf{1 9 5 1}$ & $\mathbf{1 9 5 5}$ & $\mathbf{1 9 5 9}$ & $\mathbf{1 9 6 4}$ & $\mathbf{1 9 6 6}$ & $\mathbf{1 9 7 0}$ & $\begin{array}{l}\mathbf{1 9 7 4} \\
\text { fév }\end{array}$ & $\begin{array}{l}\mathbf{1 9 7 4} \\
\text { oct }\end{array}$ & $\mathbf{1 9 7 9}$ \\
\hline $\begin{array}{l}\text { Parti } \\
\text { travailliste }\end{array}$ & 35 & 27 & 27 & 27 & 27 & 28 & 32 & 27 & 24 & 23 & 22 \\
\hline $\begin{array}{l}\text { Parti } \\
\text { conservateur }\end{array}$ & 4 & 4 & 6 & 6 & 7 & 6 & 3 & 7 & 8 & 8 & 11 \\
\hline Parti libéral & 6 & 5 & 3 & 3 & 2 & 2 & 1 & 1 & 2 & 2 & 1 \\
\hline Plaid Cymru & 0 & 0 & 0 & 0 & 0 & 0 & 0 & 0 & 2 & 3 & 2 \\
\hline Independent & & & & & & & & 1 & & & \\
\hline
\end{tabular}

Source : Données tirées de l'ouvrage de Peter JOYCE, op. cit. 